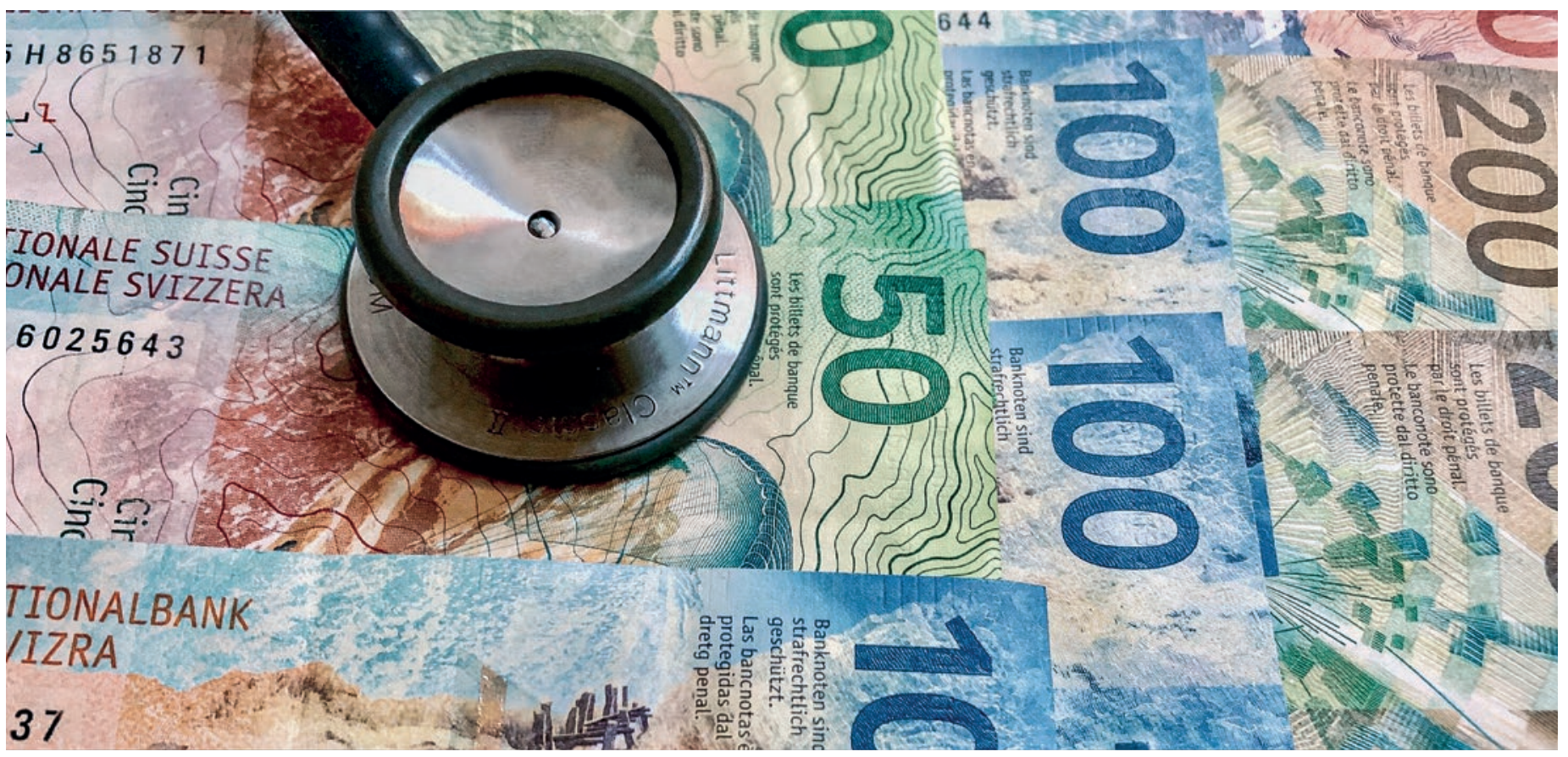

\title{
Gesundheitskosten: \\ Mythen und Fakten reloaded
}

\section{Daniel Mahler}

Dr. med., Kommission "Strategie und Kommunikation» der Schweizerischen Gesellschaft für Dermatologie und Venerologie (SGDV)

Obwohl die Schweiz weltweit eines der qualitativ besten Gesundheitswesen mit zu anderen europäischen Ländern vergleichbaren Kosten aufweist, werden politische Sparmassnahmen diskutiert, die das System gefährden. Die folgende Analyse bringt Fakten und Lösungsansätze.

Namhafte internationale Untersuchungen bestätigen, dass die Schweiz eines der weltweit besten Gesundheitssysteme aufweist [1, 2]. Der Zugang zu Behandlungen ist sehr gut, die Wartezeiten sind kurz [3]. Ein aktueller Bericht des Schweizerischen Gesundheitsobservatoriums (Obsan) zeigt, dass die Menschen mit der Qualität des Gesundheitswesens

Ein aktueller Bericht des Schweizerischen Gesundheitsobservatoriums zeigt, dass die Menschen mit der Qualität des Gesundheitswesens hochzufrieden sind.

im Vergleich mit anderen Ländern hochzufrieden sind [4]. Es ist deswegen unabdingbar, dass wir Ärztinnen und Ärzte uns dafür einsetzen, diese hohe Qualität zu erhalten. Denn in letzter Konsequenz sind wir es und nicht die politisch fachfremden Verantwortlichen, welche die aktuell diskutierten Leistungseinschränkungen gegenüber unseren Patientinnen und Patienten verantworten und auch rechtfertigen müssen.

Bis zum Jahr 2014 war die gesundheitspolitische Welt intakt: Die Gesundheitskosten, welche gerne als Relation zum Bruttoinlandprodukt (BIP) dargestellt werden, lagen damals mit 11,1\% gleichauf mit umgebenden europäischen Ländern. Bereits im Jahr darauf stieg das Verhältnis jedoch deutlich auf 11,9\% an. Das für diese Zahlen verantwortliche Bundesamt für Statistik (BfS) [5] begründete diese Veränderung unter anderem mit einer neuen Berechnungsmethode. In den Jahren 2016 und 2017 wurden 12,2 respektive 12,4\% publiziert. Daraufhin wurden politisch und medial Massnahmen gegen die angeblich überdurchschnittlich ansteigenden Kosten gefordert. 


\section{Politischer Aktionismus im Gesundheitswesen}

Es kam zu den bekannten bundesrätlichen Tarifeingriffen, welche die Sprechstundenzeit auf 20 Minuten und die Vergütung für Spezialistentätigkeiten einschränkten. Aus einem Katalog von 38 Massnahmen zur Senkung der Gesundheitskosten [6] entstanden zwei Kostendämpfungs-Massnahmenpakete, welche aktuell im Parlament diskutiert werden [7]. Die einschneidendsten Massnahmen sind die geplante Abschaffung der freien Arztwahl («Erstberatungsstelle») sowie die Einführung eines Globalbudgets («Zielvorgaben für Kosten im Gesundheitswesen»). Letztere wird als indirekter Gegenvorschlag zur CVP-Initiative «Kostenbremse im Gesundheitswesen» lanciert [8]. Auch die SP plant, mit ihrer «Prämien-Entlastungs-Initiative» die Problematik anzugehen [9].

\section{Das Narrativ der europaweit höchsten Gesundheitskosten ist falsch}

Ende Oktober 2020 hat das BfS die Daten zu den Gesundheitskosten wiederum retrospektiv um etwa einen Prozentpunkt nach unten korrigiert. Es zeigt sich,

\section{Der Anstieg der OKP-Kosten wie auch der-} jenige der Krankenkassenprämien fielen in den letzten Jahren zunehmend kleiner aus.

dass die Schweiz im Jahr 2018 bei einem Anteil von $11,2 \%$ am BIP auf Platz 4 hinter den USA, Deutschland und Frankreich weiterhin auf Augenhöhe mit anderen europäischen Ländern liegt [5]. Es ist indes richtig, dass die Gesundheitskosten jährlich gestiegen sind. Der Anstieg der OKP-Kosten wie auch derjenige der Krankenkassenprämien fielen in den letzten Jahren jedoch zunehmend kleiner aus [10-12].

\section{Nutzensteigerung im Gesundheitswesen nicht unbeachtet lassen}

Die Nutzenexplosion im Gesundheitswesen durch zunehmende medizinische Innovationen ist unbestritten [13]. Es gibt heute $40 \%$ weniger verlorene Lebensjahre durch frühe Todesfälle als 1996. Auch die Krebsmortalität ist seither um einen Drittel gesunken. In den letzten 100 Jahren stieg die Lebenserwartung von 50 auf 80 Jahre an [12], was einer der höchsten weltweit entspricht [14]. Durch zeitgemässe Behandlungen konnte bei verschiedenen Erkrankungsbildern, in der Dermatologie beispielsweise bei Psoriasis und Neurodermitis, die Lebens- qualität von Patientinnen und Patienten erheblich verbessert werden.

\section{Demographische Entwicklung und medi- zinische Innovationen als Haupttreiber}

Die demographische Entwicklung als unvermeidlicher Haupttreiber des Kostenanstiegs im Gesundheitswesen habe ich bereits 2018 in dieser Zeitung beschrieben [15]: Verantwortlich dafür sind die europaweit höchste Zuwanderungsrate ( $>1 \%$ pro Jahr) und die zunehmende Überalterung der Bevölkerung. Im Jahr 2045 wird der Anteil der >65-jährigen Menschen um über $84 \%$ zugenommen haben.

\section{Nachfrage zum Luxusgut "Gesundheit» steigt mit dem Wohlstand}

Verschiedene internationale Rankings zeigen die Schweiz hinsichtlich Lebensqualität auf den vordersten Plätzen [16]. Nicht umsonst haben wir auch kaufkraftbereinigt eines der weltweit höchsten Durchschnittseinkommen [17]. Das zunehmende durchschnittliche Haushaltsbruttoeinkommen hat in den letzten zehn Jahren dazu geführt, dass trotz prozentual gestiegener Ausgaben für Krankenkassenprämien der Sparanteil deutlich höher geworden ist [18].

\section{Steigende Gesundheitskosten betreffen Menschen mit tiefen Einkommen über- proportional}

Je nach Kanton geben vierköpfige Familien mit tieferen Einkommen für Gesundheitskosten einen Anteil von bis zu 20\% ihres Bruttoeinkommens aus [19]. Umfragen zeigen auch, dass Finanzierungsprobleme von Gesundheitsleistungen seit dem Jahr 2010 deutlich gestiegen sind [4]. Einer der hauptsächlichen Gründe dafür ist, dass der Selbstkostenanteil bei Krankheitsfällen in der Schweiz international gesehen hoch bzw. die staatliche Beteiligung an den Gesundheitskosten eher tief ist [20]. Diese Belastung muss durch sozialpolitische Massnahmen und nicht durch Leistungsbeschränkungen unter Kontrolle gebracht werden

\section{FMH und kantonale Ärztegesellschaften: Einheitliches Vorgehen der Ärzteschaft anstreben}

Die Schweizerische Ärztegesellschaft FMH, welche den schwierigen Spagat zwischen den Grundversorgern und den Spezialisten zu leisten hat, wies mehrfach 
darauf hin, dass die Ärzteschaft mit einer Stimme auftreten muss.

Um die Tarifautonomie zurückzuerhalten, müssen nachdem der Bundesrat kürzlich den TARDOC in der jetzigen Form abgelehnt hat - sämtliche Tarifpartner nun an einem Strang ziehen. Neben einem Einzelleistungstarif machen Pauschaltarife bei gut standardisierbaren interventionellen Leistungen Sinn. In der regulären Sprechstunde führen sie jedoch zu Behandlungsminimalismus und positiver Risikoselektion. Gerade für spezielle Patientengruppen wie polymorbiden Menschen oder solchen mit Behinderungen sowie für Kinder muss sich das ärztliche Engagement lohnen.

\section{Fachgesellschaften: Fokus auf Qualität reduziert Kosten}

Von Ärztinnen und Ärzten ist eine hohe Behandlungsqualität anzustreben. Die kürzlich verabschiedete KVG-Revision "Qualität und Wirtschaftlichkeit» [21] ist ein Schritt in die richtige Richtung. Sie darf aber nicht zu unverhältnismässigem Aufwand, unnötiger Büro-

\section{«Schwarze Schafe», die fachlich inkompetent arbeiten, sind durch Standeskommissionen konsequent abzumahnen.}

kratie und hohen Regulationskosten führen. Möglichkeiten dafür bestehen in der Förderung und Umsetzung von evidenzbasierten Behandlungsleitlinien und Smarter-Medicine-Kriterien [22], der Verbesserung von Fort- und Weiterbildung sowie dem fachspezifischen Erarbeiten von Qualitäts- und Hygienekonzepten. Klar ist auch: Sogenannt "schwarze Schafe», die fachlich inkompetent arbeiten, sind durch Standeskommissionen konsequent abzumahnen.

\section{Ärztinnen und Ärzte: Informieren Sie über die gesundheits- politischen Vorgänge}

Dr. med. Daniel Mahler Dermapraxis Luzern

Alpenstrasse 9

CH-6004 Luzern

Tel. 0414108383

dermapraxis-luzern[at] hin.ch
Zusammengefasst besteht heute die politische Tendenz, das freiheitliche Gesundheitssystem zunehmend zu beschneiden. Es ist unser aller Aufgabe, eine Zweiklassenmedizin in der Schweiz zu vermeiden. Unser Staat muss der Bevölkerung nicht nur ein bezahlbares, sondern in erster Linie eine qualitativ hochwertige Gesundheitsversorgung garantieren. Sprechen Sie deswegen mit Ihren Patientinnen und Patienten über die zunehmenden Missstände im schweizerischen Gesundheitswesen. Schliesslich bestimmen diese als Wählerinnen und Wähler die entsprechenden Geschicke mit.

\section{Bildnachweis}

Matthias Scholer

\section{Literatur}

1 Healthcare Access and Quality Index based on mortality from causes amenable to personal health care in 195 countries and territories, 1990-2015: a novel analysis from the Global Burden of Disease Study 2015. Lancet. 2017;390(10091):P231-66.

2 Euro Health Consumer Index 2018 Report. Health Consumer Powerhouse Ltd., 2019.

3 Kopetsch T. Facharzttermine im internationalen Vergleich: Geringe Wartezeiten in Deutschland. Dtsch Arztebl. 2015;112(31-32)

4 Pahud O. Erfahrungen der Wohnbevölkerung ab 18 Jahren mit dem Gesundheitssystem - Situation in der Schweiz und im internationalen Vergleich. Analyse des International Health Policy (IHP) Survey 2020 der amerikanischen Stiftung Commonwealth Fund (CWF) im Auftrag des Bundesamtes für Gesundheit (BAG). Neuenburg: Schweizerisches Gesundheitsobservatorium; Obsan Bericht 12/2020.

5 https://www.bfs.admin.ch/bfs/de/home/statistiken/gesundheit/ kosten-finanzierung.html

6 https://www.bag.admin.ch/bag/de/home/versicherungen/ krankenversicherung/kostendaempfung-kv.html

7 https://www.bag.admin.ch/bag/de/home/versicherungen/ krankenversicherung/krankenversicherung-revisionsprojekte. html

8 https://www.cvp.ch/de/kampagnen/initiative-fuer-tieferepraemien-kostenbremse-im-gesundheitswesen

9 https://www.sp-ps.ch/de/kampagnen/weitere-kampagnen-unddebatten/pramien-entlastungs-initiative

10 https://anneepolitique.swiss/dossiers/139-pramien-und-kostenentwicklung-in-der-krankenversicherung-2010-2020

11 Beschlussprotokoll der ersten und zweiten Ärztekammer 2020. Schweiz Ärzteztg. 2021;102:45-72.

12 Interpharma: Gesundheitswesen Schweiz - Ausgabe 2019

13 Schlup J, Wille N. Kostenexplosion? Nutzenexplosion! Synapse. 2019;3:6-8.

14 https://de.wikipedia.org/wiki/Liste_von_L\%C3\%A4ndern_nach_ durchschnittlicher_Lebenserwartung

15 Mahler D. Gesundheitskosten: Mythen und Fakten: Schweiz Ärzteztg. 2018;99:1438-40.

16 https://www.usnews.com/news/best-countries/quality-of-liferankings

17 https://de.wikipedia.org/wiki/Liste_der_L\%C3\%A4nder_nach_ Durchschnittslohn

18 https://www.swissinfo.ch/ger/haushaltsbudget das-machenschweizer-haushalte-mit-ihren-10-114-franken-monatseinkommen/46178808

19 https://www.tagesanzeiger.ch/diese-kantone-knausern-beiverbilligungen-am-meisten-915750427143

20 https://www.oecd-ilibrary.org/social-issues-migration-health/ gesundheit-auf-einen-blick_20791887 (7.6 Finanzierung der Gesundheitsversorgung)

21 https://www.bag.admin.ch/bag/de/home/versicherungen/ krankenversicherung/qualitaetsentwicklung-schweiz.html

22 https://www.smartermedicine.ch/de/home.html 